\title{
Developing Models for Type-N Coaxial VNA Calibration Kits within the NIST Microwave Uncertainty Framework*
}

\author{
Jeffrey A. Jargon, Dylan F. Williams, and Paul D. Hale \\ National Institute of Standards and Technology, 325 Broadway, M/S 672.03, Boulder, CO 80305 USA \\ Email: jeffrey.jargon@nist.gov, Tel: +1.303.497.4961
}

\begin{abstract}
We developed models for Type-N coaxial vector network analyzer (VNA) calibration kits within the NIST Microwave Uncertainty Framework. First, we created physical models of commercially-available standards that support multiline thru-reflect-line (TRL) and open-short-load-thru (OSLT) calibrations, and included error mechanisms in each of the standards' constituent parameters that were utilized in the NIST Microwave Uncertainty Framework to propagate uncertainties. Next, we created a measurement-based model of a commercial electronic calibration unit (ECU) by characterizing the scattering parameters of its internal states with a multiline TRL calibration. Finally, we calibrated a network analyzer using the three calibration methods, and compared measurements, including uncertainties, made on a number of verification devices. We show that the three calibrations agreed to within their respective uncertainties.
\end{abstract}

Index Terms - calibration, coaxial, electronic calibration unit, physical models, uncertainty, vector network analyzer, verification.

\section{INTRODUCTION}

The multiline, thru-reflect-line (TRL) calibration [1] is perhaps the most fundamental and accurate vector network analyzer (VNA) calibration for coaxial circuits. Multiline TRL calibrations measure the propagation constant of the line standards so that the characteristic impedance can be transformed to a selected reference impedance, and offer high bandwidth and accuracy through the use of multiple transmission-line standards. However, a set of coaxial lines, some relatively long, is required to obtain a wide-band measurement. Coaxial airlines also require considerable care to ensure good connections without damaging the standards. Furthermore, a set of lines can be costly, and measurements are time-consuming.

Other types of VNA calibrations make use of compact, lumped-element standards, the most common being openshort-load-thru (OSLT) and line-reflect-match (LRM) methods [2]. They provide calibration procedures that are easier to perform, oftentimes at the cost of lower accuracy.

Over the years, electronic calibration units (ECUs) have become a viable alternative to the aforementioned methods. First proposed in 1993 [3], these units provide the advantage of requiring only one connection and are capable of rapidly switching among a large variety of reflection coefficients and low-loss transmission coefficients. Recently, newer commercial units have been shown to be stable enough to be used in place of mechanical verification artifacts $[4,5]$.
In this paper, we utilize the NIST Microwave Uncertainty Framework [6-10] to develop physical models of commercially available Type-N multiline TRL and OSLT coaxial calibrations kits, and then use the multiline TRL calibration to create a traceable measurement-based model of an ECU. The NIST Microwave Uncertainty Framework utilizes parallel sensitivity and Monte-Carlo analyses, and enables us to capture and propagate the significant $S$ parameter measurement uncertainties and statistical correlations between them [11]. By identifying and modeling the physical error mechanisms in the calibration standards, we can determine the statistical correlations between both the scattering parameters at a single frequency and uncertainties at different frequencies. These uncertainties can then be propagated to measurements of the devices under test. In the following sections, we describe our methodology in further detail, and compare measurements and uncertainties made on a number of verification devices.

\section{Model DEVELoPMENT}

We began by modeling the multiline TRL calibration standards (an offset short, and five airlines of varying lengths) for purposes of determining uncertainties. Table I lists the line lengths and associated uncertainties for the multiline TRL standards, and Table II lists the other sources of uncertainty for the standards. Our values and distributions of the uncertainties come from a variety of sources, including manufacturers' specifications and an IEEE standard [12].

The NIST Microwave Uncertainty Framework was employed to construct models for the calibration standards. The airline and offset-short standards were modeled with closed-form expressions for coaxial lines of finite metal conductivity [13]. The framework was also used for automatically propagating the uncertainties to the calibrated verification devices in conjunction with the calibration engine, StatistiCAL ${ }^{\mathrm{TM}}[14,15]$, which utilizes a "mix-and-match" philosophy to VNA calibrations.

Next, the OSLT standards were modeled with the values and uncertainties listed in Tables II and III. We modeled the load standard as a simple $50 \mathrm{Ohm}$ resistor after observing that the magnitudes of the measured reflection coefficients for both the male and female connectors were less than $-30 \mathrm{~dB}$ at most frequencies. The offset lengths of the open and short standards 
were estimated from the respective phase delays measured with the multiline TRL calibration.

Finally, we created a measurement-based model of our ECU by characterizing the $S$-parameters of its internal states with a multiline TRL calibration. The added complication here was that our airlines had male connectors on both ports, so a male-to-male adapter was required to measure the insertable ECU. We then de-embedded the calibrated adapter to properly characterize the ECU.

Table I. Lengths and uncertainties of the Type-N TRL standards.

\begin{tabular}{|c|c|}
\hline Line Designation & $\begin{array}{c}\text { Length (mm) } \pm \text { Uncertainty } \\
\text { (Distribution) }\end{array}$ \\
& \\
\hline & $36.966 \pm 0.005$ (Rectangular) \\
Airline 1 & $43.472 \pm 0.005$ (Rectangular) \\
Airline 2 & $49.959 \pm 0.005$ (Rectangular) \\
Airline 3 & $56.442 \pm 0.005$ (Rectangular) \\
Airline 4 & $99.904 \pm 0.005$ (Rectangular) \\
Airline 5 & $18.955 \pm 0.005$ (Rectangular) \\
Offset Short & \\
\hline
\end{tabular}

Table II. Physical error mechanisms of the Type-N standards.

\begin{tabular}{|c|c|}
\hline Mechanism (units) & $\begin{array}{c}\text { Value } \pm \text { Uncertainty } \\
\text { (Distribution) }\end{array}$ \\
& \\
\hline & \\
Inner Cond. Diameter $(\mathrm{mm})$ & $3.04 \pm 0.0026$ (Rectangular) \\
Outer Cond. Diameter $(\mathrm{mm})$ & $7.0 \pm 0.0051$ (Rectangular) \\
Pin Diameter $(\mathrm{mm})$ & $1.651 \pm 0.0127$ (Rectangular) \\
Pin Depth (mm) & $0.051 \pm 0.051$ (Rectangular) \\
Metal Conductivity (S/m) & $7.9 \times 10^{6} \pm 4 \times 10^{6}$ (Rectangular) \\
Relative Dielectric Constant & $1.000535 \pm 0$ \\
Dielectric Loss Tangent & $0 \pm 0$ \\
& \\
\hline
\end{tabular}

Table III. Physical error mechanisms of the Type-N OSLT standards.

\begin{tabular}{|c|c|}
\hline Mechanism (units) & $\begin{array}{c}\text { Value } \pm \text { Uncertainty } \\
\text { (Distribution) }\end{array}$ \\
& \\
\hline & \\
Male Open Offset Length $(\mathrm{mm})$ & $6.504 \pm 0.005$ (Rectangular) \\
Female Open Offset Length $(\mathrm{mm})$ & $1.944 \pm 0.005$ (Rectangular) \\
Open Conductance $(1 / \Omega)$ & $0 \pm 0$ \\
Open Capacitance $(\mathrm{pF})$ & $0 \pm 0$ \\
Male Short Offset Length $(\mathrm{mm})$ & $5.321 \pm 0.005$ (Rectangular) \\
Female Short Offset Length $(\mathrm{mm})$ & $0.000 \pm 0.005$ (Rectangular) \\
Short Resistance $(\Omega)$ & $0 \pm 0$ \\
Short Inductance $(\mathrm{nH})$ & $0 \pm 0$ \\
Load Resistance $(\Omega)$ & $50.0 \pm 0.1$ (Rectangular) \\
Load Inductance $(\mathrm{nH})$ & $0.0 \pm 0.1$ (Rectangular) \\
& \\
\hline
\end{tabular}

\section{MEASUREMENT COMPARISON}

Once the multiline TRL, OSLT, and electronic calibration standards were defined, we used the three sets of standards to calibrate the measurements of verification devices for comparison purposes. Once again, for the TRL calibration, the calibrated adapter was required for measuring the insertable devices. Figures 1-5 show calibrated S-parameters and corresponding $95 \%$ confidence bounds calculated from the sensitivity analysis performed in the NIST Uncertainty Framework for a $20 \mathrm{~dB}$ attenuator, a $50 \mathrm{~dB}$ attenuator, an airline, and a Beatty line. Dashed curves in the figures correspond to confidence bounds.

In each of the figures, the three calibrated measurements agreed to within their respective uncertainties at most frequencies, although the OSLT-calibrated measurements were visibly noisier. The uncertainties of the OSLT-calibrated measurements were also larger in general. For instance, the mean confidence intervals for $\left|S_{21}\right|$ of the 20-dB attenuator were approximately $\pm 0.07 \mathrm{~dB}$ with multiline TRL, $\pm 0.15 \mathrm{~dB}$ with OSLT, and $\pm 0.07 \mathrm{~dB}$ with the ECU.

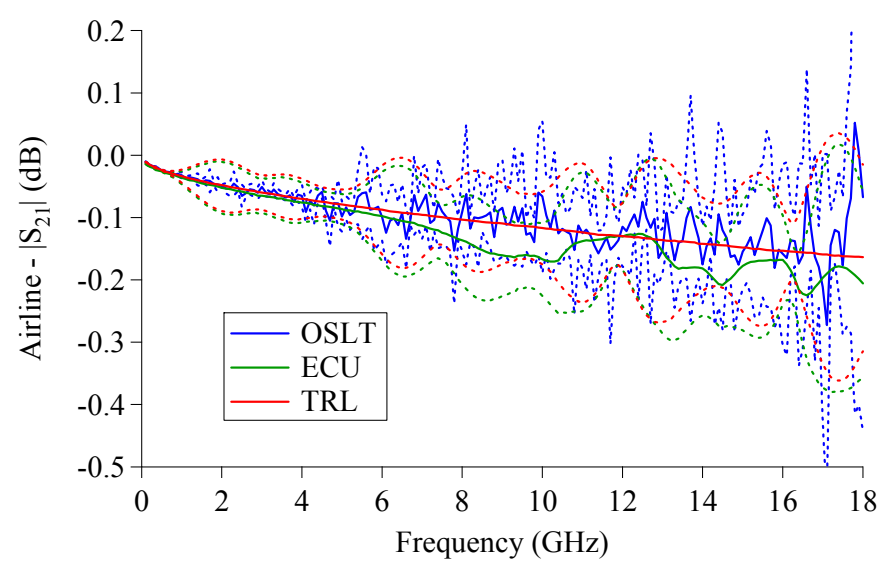

Fig. 1. Comparing measurements and 95\% confidence intervals of the airline's transmission coefficients.

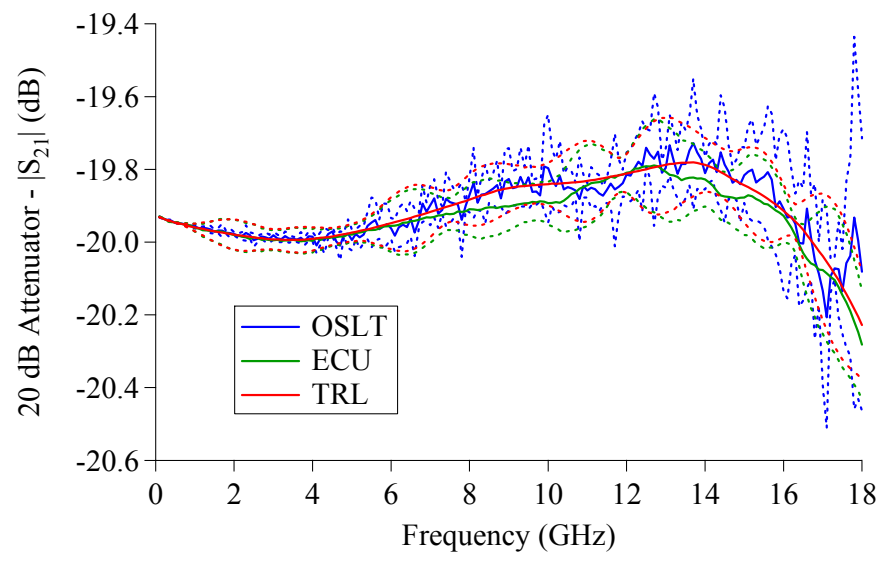

Fig. 2. Comparing measurements and $95 \%$ confidence intervals of the $20 \mathrm{~dB}$ attenuator's transmission coefficients. 


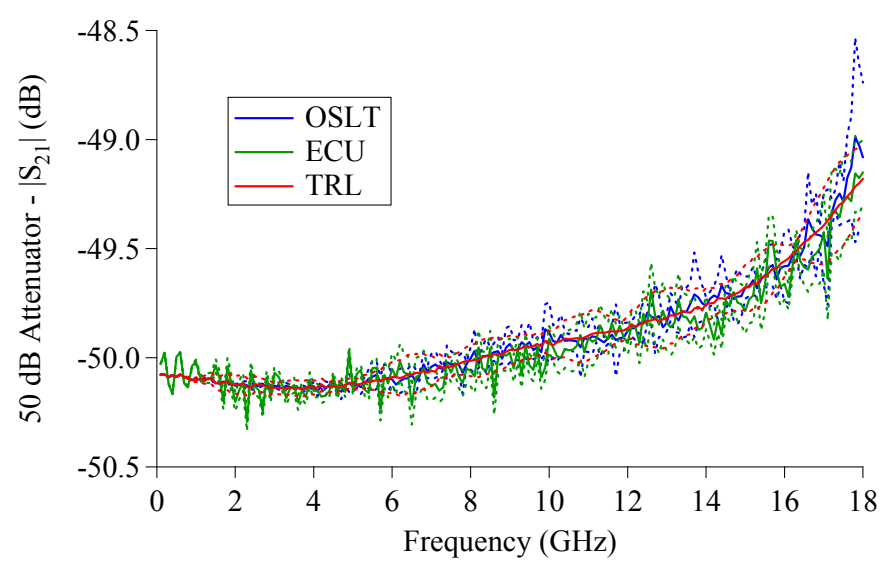

Fig. 3. Comparing measurements and 95\% confidence intervals of the $50 \mathrm{~dB}$ attenuator's transmission coefficients.

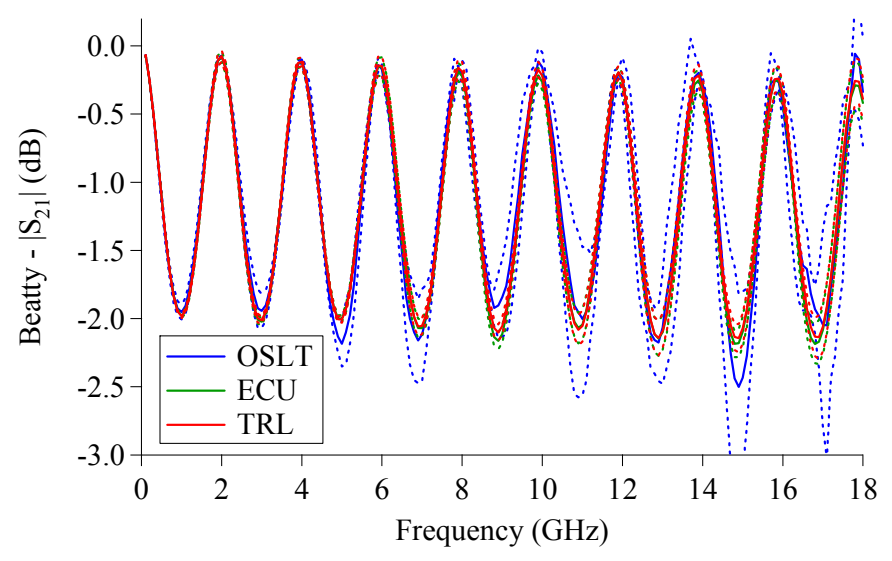

Fig. 4. Comparing measurements and 95\% confidence intervals of the Beatty line's transmission coefficients.

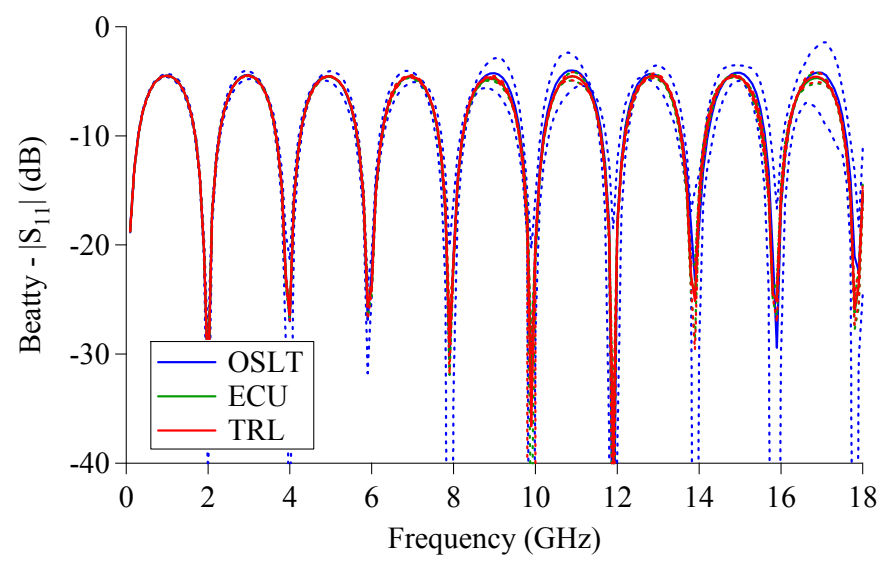

Fig. 5. Comparing measurements and 95\% confidence intervals of the Beatty line's reflection coefficients.

\section{CONCLUSIONS}

We have developed physical and measurement-based models of Type $\mathrm{N}$ coaxial calibration kits for vector network analyzers that support multiline TRL, OSLT, and electronic calibrations within the NIST Microwave Uncertainty Framework. The verification devices measured with the three calibration approaches agree to within their respective uncertainties. Although other sources of uncertainty may be included in a final uncertainty analysis, we believe these minor additions will not significantly affect the overall uncertainties.

The principle advantage of characterizing an ECU and providing uncertainties is that the unit can be used as a working set of standards that requires only a single connection to calibrate the VNA, saving both time and wear-and-tear on the VNA test ports as well as the TRL and OSLT standards.

\section{ACKNOWLEDGEMENT}

*This work was supported by the U.S. Department of Commerce, and is not subject to U.S. copyright. The authors thank Ronald Ginley for the use of his multiline TRL calibration kit, and David Walker for the use of his verification kit.

\section{REFERENCES}

[1] R. B. Marks, "A multiline method of network analyzer calibration," IEEE Trans. Microwave Theory Tech., vol. 39, no. 7, pp. 1205-1215, July 1991.

[2] D. K. Rytting, "Network analyzer error models and calibration methods," 52nd ARFTG Conference, Short Course on Computer-Aided RF and Microwave Testing and Design, Dec. 1998.

[3] V. Adamian, "A novel procedure for network analyzer calibration and verification," 41st ARFTG Conference, Spring 1993.

[4] D. F. Williams, A. Lewandowski, D. LeGolvan and R. Ginley, "Electronic vector-network-analyzer verification," IEEE Microwave Magazine, pp. 118-123, Oct. 2009.

[5] D. F. Williams, A. Lewandowski, D. LeGolvan, R. Ginley, C. M. Wang and J. Splett, "Use of electronic calibration units for vector-network-analyzer verification," 74th ARFTG Conference, Dec. 2009.

[6] D. F. Williams, NIST Microwave Uncertainty Framework, Beta Version, software.cfm, 2015.

[7] J. A. Jargon, D. F. Williams, T. M. Wallis, D. X. LeGolvan, and P. D. Hale, "Establishing traceability of an electronic calibration unit using the NIST Microwave Uncertainty Framework," 79th ARFTG Microwave Measurement Conference, Montreal, CANADA, Jun. 2012.

[8] J. A. Jargon, U. Arz, and D. F. Williams, "Characterizing WR-8 waveguide-to-CPW probes using two methods implemented within the NIST Uncertainty Framework," 80th ARFTG Microwave Measurement Conference, San Diego, CA, Nov. 2012. 
[9] J. A. Jargon, D. F. Williams, P. D. Hale, and M. D. Janezic, "Characterizing a noninsertable directional device using the NIST Uncertainty Framework," 83rd ARFTG Microwave Measurement Conference, Tampa Bay, FL, Jun. 2014.

[10] J. A. Jargon, C. H. Cho, D. F. Williams, and P. D. Hale, "Physical models for $2.4 \mathrm{~mm}$ and $3.5 \mathrm{~mm}$ coaxial VNA calibration kits developed within the NIST Microwave Uncertainty Framework," 85th ARFTG Microwave Measurement Conference, Phoenix, AZ, May 2015.

[11] A. Lewandowski, D. F. Williams, P. D. Hale, C. M. Wang, and A. Dienstfrey, "Covariance-matrix-based vector-networkanalyzer uncertainty analysis for time-and frequency-domain measurements," IEEE Trans. Microwave Theory Tech., vol. 58, no. 7, pp. 1877-1886, July 2010.
[12] IEEE Standard 287-2007 - Standard for precision coaxial connectors (DC to $110 \mathrm{GHz}$ ).

[13] A. Lewandowski, "Multi-frequency approach to vector-network analyzer scattering-parameter measurements," Ph.D. Thesis, Warsaw University of Technology, 2010.

[14] D. F. Williams, StatistiCAL VNA Calibration Software Package, $\quad$ http://www.nist.gov/ctl/rf-technology/relatedsoftware.cfm, 2015.

[15] D.F. Williams, C.M. Wang, and U. Arz, "An optimal vectornetwork-analyzer calibration algorithm," IEEE Trans. Microwave Theory and Tech., vol. 51, no. 12, pp. 2391-2401, Dec. 2003. 